\title{
A Note on Translation and Transcription
}

I conducted all of the interviews for this study in either IsiZulu or English, depending on the preference and ability of each interviewee. All translations from IsiZulu to English are my own, unless otherwise noted. In the transcriptions I have retained IsiZulu words for significant concepts or categories that appear repeatedly throughout the book, or where particular words deserve scrutiny for other reasons. Some of the transcriptions reflect editorial changes that I made during the process of translation in order to enhance clarity and flow. 
This page intentionally left blank 